\title{
Eruptive xanthomas in a patient with severe hypertriglyceridemia and type 2 diabetes
}

\author{
Barry Ladizinski MD, Kachiu C. Lee MD MPH
}

Competing interests: None declared.

This article has been peer reviewed.

Affiliations: Johns Hopkins School of Public Health (Ladizinski), Baltimore, Md.; Department of Dermatology (Lee), Brown University,

Providence, RI

Correspondence to:

Kachiu C. Lee,

kachiu@gmail.com

CMAJ 2013. DOI:10.1503 /cmaj.130148
1 46-year-old man with obesity, hypertension, hyperlipidemia and bipolar disorder presented with a rash (Figure 1) accompanied by ongoing excessive urine production, excessive thirst and blurred vision. The lesions had appeared on his arms 1 month earlier and had spread to his neck, buttocks and legs. He was taking quetiapine and metoprolol. Both of the patient's parents had type 2 diabetes mellitus.

A physical examination showed crops of firm yellow-red papules (diameter 1-3 $\mathrm{mm}$ ) distributed on the patient's neck, bilateral extremities and buttocks, suggestive of eruptive xanthomas. Laboratory investigations showed elevated levels of triglycerides (64.2 [normal $0.6-2.8] \mathrm{mmol} / \mathrm{L})$, cholesterol (18.2 [normal 3.1-5.2] mmol/L), acetylated hemoglobin (139.3 [normal 25.6-42.0] $\mathrm{mmol} / \mathrm{mol}$ ) and glucose (31.2 [normal 3.3-6.1] $\mathrm{mmol} / \mathrm{L}$ ). Results of kidney and liver function tests were normal, as were the results of tests for thyroid stimulating hormone, triiodothyronin, thyroxin, amylase and lipase.

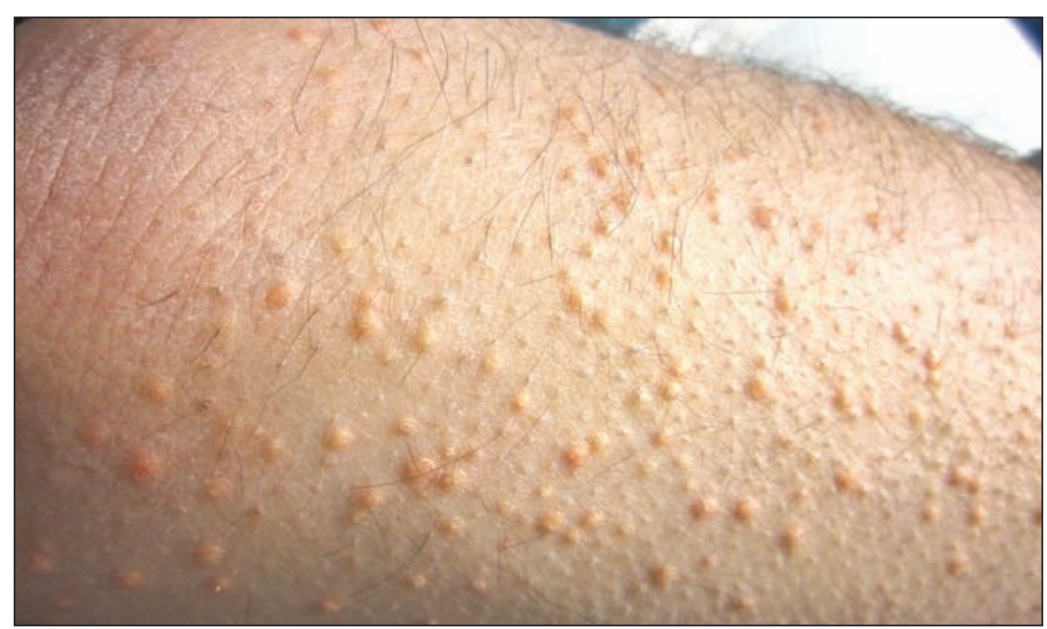

Figure 1: Firm, yellow, dome-shaped papules on the arm of a 46-year-old man with hypertriglyceridemia, hypercholesterolemia and hyperglycemia.
Eruptive xanthomas are characterized by the sudden appearance of grouped, yellow-red papules scattered over the trunk, arms, legs and buttocks. ${ }^{1}$ The condition is associated with the markedly elevated serum triglyceride levels that occur with hyperlipidemia syndromes (ie., Fredrickson-Levy types I, IV and V) or with diabetes mellitus, hypothyroidism, obesity, pancreatitis, nephrotic syndrome, cholestatic liver disease, dysglobulinemia and as an adverse effect of using certain medications (e.g., estrogens, corticosteroids, systemic retinoid agents). ${ }^{1,2}$ Treatment involves management of the underlying condition, whereby lesions resolve within weeks to months. ${ }^{1,3}$ Patients should also be counseled on lifestyle changes, including weight control, adopting a lowfat diet, exercising and quitting smoking. ${ }^{3}$

Our patient's lesions were likely due to a combination of type $\mathrm{V}$ hyperlipoproteinemia (mixed hypertriglyceridemia), undiagnosed type 2 diabetes mellitus and use of antipsychotic agents, although it is difficult to determine the instigating factor. He was admitted to the intensive care unit, and his condition responded well to treatment with insulin, metformin and gemfibrozil. The patient's quetiapine was stopped, and he was transitioned to topiramate without complications. The patient's triglyceride levels improved to 13.8 $\mathrm{mmol} / \mathrm{L}$ after 8 days of treatment, and his skin lesions improved after 8 weeks.

\section{References}

1. Parker F. Xanthomas and hyperlipidemias. J Am Acad Dermatol 1985; $13: 1-30$

2. Robson KJ, Piette WW. Cutaneous manifestations of systemic diseases. Med Clin North Am 1998;82:1359-79.

3. National Cholesterol Education Program (NCEP) Expert Panel on Detection, Evaluation, and Treatment of High Blood Cholesterol in Adults (Adult Treatment Panel III). Third report of the National Cholesterol Education Program (NCEP) Expert Panel on Detection, Evaluation, and Treatment of High Blood Cholesterol in Adults (Adult Treatment Panel III) final report. Circulation 2002;106:3143-421. 